\title{
The metabolic activity of gut microbiota in obese children is increased compared with normal-weight children and exhibits more exhaustive substrate utilization
}

\author{
AN Payne ${ }^{1}$, C Chassard $^{1}$, M Zimmermann ${ }^{1}$, P Müller $^{2}$, S Stinca ${ }^{1}$ and C Lacroix ${ }^{1}$ \\ ${ }^{1}$ Laboratory of Human Nutrition, Institute of Food, Nutrition and Health, ETH Zürich, Zürich, Switzerland and \\ ${ }^{2}$ Ostschweizer Kinderspital, St. Gallen, Switzerland
}

Objective: The gut microbiota contribute otherwise impossible metabolic functions to the human host. Shifts in the relative proportions of gut microbial communities in adults have been correlated with intestinal disease and have been associated with obesity. The aim of this study was to elucidate differences in gut microbial compositions and metabolite concentrations of obese versus normal-weight children.

Materials and methods: Fecal samples were obtained from obese $(n=15$; mean body mass index $(\mathrm{BMI})$ s.d. score $=1.95)$ and normal-weight $(n=15$; BMI s.d. score $=-0.14)$ Swiss children aged 8-14 years. Composition and diversity of gut microbiota were analyzed by $\mathrm{qPCR}$ and temperature gradient gel electrophoresis (TGGE).

Results: No significant quantitative differences in gut microbiota communities of obese and normal-weight children were identified. Microbial community profiling by TGGE revealed a high degree of both intra- and intergroup variation. Intergroup comparison of TGGE profiles failed to identify any distinct populations exclusive to either obese or normal-weight children. High-pressure liquid chromatography analysis identified significantly higher $(P<0.05)$ concentrations of short-chain fatty acids (SCFA) butyrate and propionate in obese versus normal-weight children. Significantly lower concentrations of intermediate metabolites were detected in obese children, suggesting exhaustive substrate utilization by obese gut microbiota.

Conclusions: Our results indicate that a dysbiosis may be involved in the etiology of childhood obesity. In turn, aberrant and overactive metabolic activity within the intestine could dictate survival or loss of individual microbial communities, leading to the altered population ratios previously identified in adult obesity.

Nutrition and Diabetes (2011) 1, e12; doi:10.1038/nutd.2011.8; published online 18 July 2011

Keywords: obesity; pediatric; short-chain fatty acid; gut microbiota; metabolism

\section{Introduction}

The human gut is home to over 1 trillion microbes, collectively referred to as the gut microbiota. Dominated by anaerobic bacteria, the gut microbiota provide essential metabolic capacities to humans, negating the necessity for human evolution of these traits. Maintenance of the delicate gut microbiota balance creates a beneficial symbiotic relationship responsible for dietary energy extraction, attenuation of infection and inflammation, as well as a

Correspondence: $\operatorname{Dr} C$ Lacroix, Laboratory of Human Nutrition, Institute of Food, Nutrition and Health, ETH Zürich, Schmelzbergstrasse 7, 8092 Zürich, Switzerland.

E-mail: christophe.lacroix@ilw.agrl.ethz.ch

Received 20 January 2011; revised 8 April 2011; accepted 23 May 2011 multitude of other processes. ${ }^{1,2} 16 \mathrm{~S}$ rRNA-Based sequencing techniques have implied changes to this delicate balance of gut microbiota, which may potentially contribute to development of several pathological conditions (e.g., irritable bowel disease) or metabolic diseases such as obesity. ${ }^{3-6}$ About $90 \%$ of the gut microbial community is dominated by members of the Bacteroidetes and Firmicutes phyla, and an increased ratio of Firmicutes to Bacteroidetes has been implicated in the development of adult obesity. ${ }^{4-10}$ Other attempts in correlating obesity to changes in individual gut microbiota communities have been unsuccessful, countering that metabolic activity and not composition of the gut microbiota might be more relevant in the development of obesity. ${ }^{10,11}$ Although the definitive contribution of the gut microbiota to obesity remains largely debated, metabolic analyses of obese and normal-weight adult feces have indeed 
identified higher short-chain fatty acid (SCFA) concentrations in obese individuals. ${ }^{11}$ Microbial fermentation of hydrolyzed polysaccharides in the large intestine results in production of SCFA acetate, propionate and butyrate; branched chain fatty acids; lactate, formate, ethanol and mixed gases (e.g., $\mathrm{CH}_{4}, \mathrm{CO}_{2}$ and $\mathrm{H}_{2}$ ). ${ }^{12}$ SCFA resulting from colonic fermentation may provide an estimated additional $10 \%$ daily dietary energy to the host, which may be used for de novo hepatic triglyceride and glucose synthesis. ${ }^{13-16}$ Hence, a mere daily energy increase of $1 \%$, providing an additional $20 \mathrm{kcal}$ per day, based on a $2000 \mathrm{kcal}$ per day diet, could result in nearly $1 \mathrm{~kg}$ of weight gain annually.

The aim of this study was to examine the gut microbial compositions and fecal metabolite concentrations of obese and normal-weight children. Fecal samples were obtained from obese ( $n=15$; mean body mass index (BMI) s.d. score $=1.95)$ and normal-weight $(n=15$; mean BMI s.d. score $=-0.14$ ) Swiss children aged $8-14$ years and analyzed for differences in both community composition and metabolite concentrations. Individual microbial populations were quantitatively evaluated by qPCR. Temperature gradient gel electrophoresis (TGGE) was used to profile the intra- and intergroup diversity of obese and normal-weight gut microbiota. Fecal metabolite concentrations were measured using high-pressure liquid chromatography (HPLC).

\section{Materials and methods}

\section{Study population}

The primary interest of our study was to generate a random cohort of obese and normal-weight children from their daily, routine environments. Obese $(n=15$; mean BMI s.d. score $=1.95)$ and normal-weight $(n=15$; mean BMI s.d. score $=-0.14$ ) Swiss children aged 8-14 years, who had not been exposed to antibiotic treatment for the past 3 months, were recruited for this study. The children were classified as obese or normal-weight, based on gender- and age-specific BMI percentiles from the Centers for Disease Control (http:// www.cdc.gov), in which obesity is defined as $\geqslant 95$ th percentile and normal-weight defined as $<85$ th percentile. The obese children were recruited from the obesity counseling center at the Ostschweizer Kinderspital in St. Gallen, Switzerland and included nine boys and six girls (Table 1). Normal-weight children were recruited from primary schools

Table 1 Characteristics of obese and normal-weight children in this study

\begin{tabular}{|c|c|c|}
\hline & Obese & Normal-weight \\
\hline Subjects (male/female) & $15(9 / 6)$ & $15(8 / 7)$ \\
\hline Age (years) & $10.6 \pm 1.3$ & $10.0 \pm 1.4$ \\
\hline $\mathrm{BMI}\left(\mathrm{kg} \mathrm{m}^{-2}\right)$ & $25.5 \pm 3.3$ & $16.8 \pm 1.8$ \\
\hline BMI s.d. score & $1.95 \pm 0.3$ & $-0.14 \pm 0.7$ \\
\hline
\end{tabular}

Abbreviations: BMI, body mass index; s.d., standard deviation. Data are shown as mean \pm s.e. in the area and included eight boys and seven girls (Table 1). All the children were Caucasian and long-term residents of Switzerland. Dietary intakes were not controlled for in this study. A brief baseline assessment of dietary intakes was performed using a short dietary questionnaire. Children in both groups were determined to consume comparable diets typical of northern Switzerland. Children's diets in this area typically contain $34-36 \%$ of energy as fat, $14-16 \%$ of energy as protein, with the main energy sources being bread, dairy and meat products. ${ }^{17}$ No food allergies were reported in either group and only two children reported altered eating habits with one obese child never consuming fish and one normal-weight child abstaining from dairy products. Lifestyle data pertaining to physical activity was not obtained. The parents of the children gave informed written consent, and the children assented to the study. The study was approved by both the ETH (EK 2009-N-01) and the canton of St. Gallen Ethics Committees (EKSG09/157/1B).

\section{Fecal sample collection and storage}

Fresh fecal samples were deposited in sterile plastic containers (Dutscher SA, Brumath, France). Containers were maintained under anaerobiosis by use of anaerobic sacks (Anaerocult A mini, Merck KGaA, Darmstadt, Germany). Fecal samples were delivered to the laboratory and aliquoted into sterile Eppendorf tubes (Vaudaux-Eppendorf AG, Basel, Switzerland) before storage at $-80^{\circ} \mathrm{C}$. The entire process from defecation to storage did not exceed $2 \mathrm{~h}$.

\section{Bacterial strains and culture conditions}

Roseburia intestinalis (DSM 14610) and Blautia hansenii (DSM 20583) were purchased from German Collection of Microorganisms and Cell Cultures (DSMZ, Braunschweig, Germany). Escherichia coli (ATCC 25288), Bacteroides fragilis (ATCC 25285T), Bifidobacterium longum (ATCC 15707) and Lactobacillus rhamnosus GG (ATCC 53103) were obtained from American Type Culture Collection (ATCC; Manassas, VA, USA). Anaerobic culture methods were used with $\mathrm{O}_{2}$-free $\mathrm{CO}_{2}$-sparged Hungate tubes sealed with butyl-rubber stoppers (Dutscher SA, Brumath, France) for cultivation of R. intestinalis, B. hansenii, B. fragilis, B. longum and L. GG. ${ }^{18}$ Strains were grown at $37^{\circ} \mathrm{C}$ in $10 \mathrm{ml}$ yeast extract-casitonefatty acid media supplemented with $2 \%$ each D-glucose and soluble starch (Sigma, Buchs, Switzerland) as previously described. ${ }^{19}$ E. coli was grown aerobically overnight at $37^{\circ} \mathrm{C}$ in Luria-Bertani broth.

\section{Nucleic acid extraction}

DNA was extracted from $250 \mathrm{mg}$ feces using the FastDNA Spin Kit for Soil (Qbiogene AG, Basel, Switzerland) and quantified using the Nanodrop ND-1000 spectrophotometer (Witec AG, Littau, Switzerland) at $260 \mathrm{~nm}$. 
Table 2 Group and species-specific 16S rRNA gene-targeted primers used in this study

\begin{tabular}{|c|c|c|c|c|}
\hline Target organism & $5^{\prime}-3^{\prime}$ Sequence & Primer & Standard & Reference \\
\hline \multirow[t]{2}{*}{ All bacteria } & ACTCCTACGGGAGGCAGCAG & Eub $338 \mathrm{~F}$ & pLME21 & 41 \\
\hline & ATTACCGCGGCTGCTGG & Eub $518 \mathrm{R}$ & & \\
\hline \multirow[t]{2}{*}{ Bacteroides } & GAAGGTCCCCCACATTG & Bac303F & B. thetaiotaomicron $16 \mathrm{~S}$ rRNA & 42 \\
\hline & CGCKACTTGGCTGGTTCAG & Bfr-Femrev & & \\
\hline \multirow[t]{2}{*}{ Firmicutes } & GGAGYATGTGGTTTAATTCGAAGCA & Firm934F & R. intestinalis $16 \mathrm{~S} \mathrm{rRNA}$ & 41 \\
\hline & AGCTGACGACAACCATGCAC & Firm1060R & & \\
\hline \multirow[t]{2}{*}{ Roseburia/E. rectale } & GCGGTRCGGCAAGTCTGA & RrecF & R. intestinalis $16 \mathrm{~S} \mathrm{rRNA}$ & 42 \\
\hline & ССТССGАСАСТСТАGTMСGАС & Rrec630mR & & \\
\hline \multirow[t]{2}{*}{ Lactobacillus } & AGCAGTAGGGAATCTTCCA & F_Lacto 05 & L. delbrueckii 16S rRNA & 43 \\
\hline & СGCСACTGGTGTTCYTCCATATA & R_Lacto 04 & & \\
\hline \multirow{2}{*}{$\begin{array}{l}\text { Bifidobacterium } \\
\text { phosphoketolase }\end{array}$} & ATCTTCGGACCBGAYGAGAC & $x f p-f w$ & xfp amplicon & 44 \\
\hline & CGATVACGTGVACGAAGGAC & $x f p-r v$ & & \\
\hline \multirow[t]{2}{*}{ Enterobacteriaceae } & САTTGACGTTACCCGCAGAAGAAGC & Eco1457F & E.coli 16S rRNA & 45 \\
\hline & СTCTACGAGACTCAAGCTTGC & Eco1652R & & \\
\hline \multirow[t]{2}{*}{ F. prausnitzii } & GGAGGAAGAAGGTCTTCGG & FPR-2F & F. prausnitzii $16 \mathrm{~S}$ rRNA & 42 \\
\hline & ААТTCСGССТАССТСТССАСТ & Fprau645R & & \\
\hline \multirow[t]{2}{*}{ Sulfate-reducing bacteria } & CGGCGTTGCGCATTTYCAYACVVT & dsrA_290F & D. piger dsrA & 46 \\
\hline & GCCGGACGATGCAGHTCRTCCTGRWA & dsrA_660R & & \\
\hline
\end{tabular}

\section{Quantitative PCR analysis}

Amplification and detection of DNA by qPCR was performed with a 7500 Fast Real-Time PCR System (Applied Biosystems Europe BV, Zug, Switzerland) using optical-grade 96-well plates. Duplicate sample analysis was routinely performed in a total volume of $25 \mu \mathrm{l}$ using SYBR Green PCR Master Mix (Applied Biosystems) containing $200 \mathrm{~nm}$ of both forward and reverse primers (Table 2). Standard curves were routinely performed for each qPCR run using serial dilutions of control DNA (Table 2). PCR conditions consisted of initial activation at $95^{\circ} \mathrm{C}$ for $10 \mathrm{~min}$; 40 cycles of denaturation at $95^{\circ} \mathrm{C}$ for $15 \mathrm{~s}$, annealing at $60^{\circ} \mathrm{C}$ for $30 \mathrm{~s}$ and elongation at $60^{\circ} \mathrm{C}$ for $30 \mathrm{~s}$. Data from duplicate samples were analyzed using the Sequence Detection Software Version 1.4 (Applied Biosystems).

\section{PCR Amplification of $16 S$ rRNA}

DNA (100 ng $\mu^{-1}$ ) was used to PCR amplify the variable V2-V3 16S rRNA gene sequence using universal primers HDA-1GC (CGCCCGGGGCGCGCCCCGGGCGGGGCGGG GGCACGG GGGGACTCCTACGGGAGGCAGCAGT) and HDA-2 (GTATTACCGCGGCTGCTGGCAC) and a modified protocol of Ogier et al. ${ }^{20}$ PCR reactions consisted of $2 \times$ Fermentas PCR Mastermix $\left(1 \times\right.$ PCR buffer, $2 \mathrm{mM} \mathrm{MgCl}_{2}$, $0.2 \mathrm{mM}$ each dNTP and $1 \mathrm{U}$ Taq polymerase) diluted 1:1 with sterile ultra-pure water (Millipore AG, Zug, Switzerland). Samples were amplified on a Biometra Personal Cycler (Biometra, Châtel-St.Denis, Switzerland). Reaction conditions were as follows: $94{ }^{\circ} \mathrm{C}$ for $5 \mathrm{~min}$; 35 cycles of $94^{\circ} \mathrm{C}$ for $3 \mathrm{~min}, 58^{\circ} \mathrm{C}$ for $30 \mathrm{~s}, 68^{\circ} \mathrm{C}$ for $1 \mathrm{~min}$ and finally $68^{\circ} \mathrm{C}$ for 7 min.

\section{TGGE analysis of PCR amplicons}

TGGE gels $(16 \mathrm{~cm} \times 16 \mathrm{~cm} \times 1 \mathrm{~mm})$ were composed of $6 \%$ acrylamide/bis-acrylamide (37.5:1). (Sigma), 7 M urea (Sigma) and $1.5 \times$ Tris-acetate-EDTA buffer. $^{21}$ TGGE was performed with $50 \mathrm{ng} 16 \mathrm{~S}$ rRNA PCR amplicons using a Dcode universal mutation system (Bio-Rad, Reinach, Switzerland). A custom marker was created by mixing equal concentrations of $16 \mathrm{~S}$ rRNA PCR amplicons of $B$. fragilis, E. coli, R. intestinalis, $B$. hansenii, B. longum and L. rhamnosus GG. Electrophoresis conditions included a pre-run of $20 \mathrm{~min}$ at $20 \mathrm{~V}$ followed by $16 \mathrm{~h}$ at $70 \mathrm{~V}$ in $1.5 \times$ Tris-acetate-EDTA buffer. Temperature was increased from $66^{\circ} \mathrm{C}$ to $70{ }^{\circ} \mathrm{C}$ using a ramp rate of $0.4^{\circ} \mathrm{Cmin}^{-1}$. Gels were stained for $30 \mathrm{~min}$ in ethidium bromide and destained for $1 \mathrm{~h}$ in $\mathrm{dH}_{2} \mathrm{O}$ before imaging.

Metabolite analysis by high-pressure liquid chromatography SCFA, branched chain fatty acids and intermediate metabolites were determined by HPLC as previously described. ${ }^{22}$ Mean metabolite concentrations of fecal samples were calculated from duplicate sample analysis.

\section{Statistical analysis}

Mean copy numbers were transformed to their base 10 logarithmic values for variance homogeneity. One-way analysis of variance was performed to test effects of weight status on bacterial populations and SCFA production using JMP 8.0 (SAS Institute Inc., Cary, NC, USA). When significant differences were found below $P<0.05$, treatment means were compared using the Tukey-Kramer HSD test. 


\section{Results}

Comparative analysis of gut microbiota communities in obese and normal-weight children

qPCR was performed to quantitatively assess individual gut microbial populations in obese and normal-weight children. Numerical variations in population numbers measured between obese and normal-weight children were not statistically significant for any population tested (Table 3). Comparative analysis of both Firmicutes and Bacteroides, a major genus of the Bacteroidetes phylum, indicated no correlation between an elevated Firmicutes/Bacteroidetes ratio and childhood obesity. The Firmicutes/Bacteroides ratio was nearly $1: 1$ in obese children, whereas normal-weight children demonstrated a higher Bacteroides/Firmicutes ratio. The butyrate-producing Roseburia/E. rectale population of Clostridia cluster XIVa was over 1 log higher in both obese and normal-weight children compared with the cluster IV butyrate-producer F. prausnitzii (Table 3). Sulfate-reducing bacteria (SRB) displayed the highest level of individual variation demonstrated by the high s.d.'s of 1.4 and 1.3 for obese and normal-weight children, respectively, (Table 3) and with individual populations ranging from $<3.0 \log _{10}$ copies $-1 \mathrm{~g}$ to $8.0 \log _{10}$ copies- $1 \mathrm{~g}$.

\section{Microbial community profiling of obese and normal-weight children}

Microbial community profiles of each obese and normalweight volunteer were assessed by TGGE (Supplementary Figures 1 and 2). TGGE is routinely used to analyze the diversity of complex biological samples by identifying $16 \mathrm{~S}$ rRNA sequence heterogeneities. Visual inspection of TGGE profiles of both obese and normal-weight children revealed an obvious and highly variable microbial diversity between volunteers, illustrated by the highly random banding patterns of each individual microbiota (Supplementary Figures 1 and 2). Samples from a small number of volunteers were difficult to resolve by TGGE, despite multiple DNA extractions and PCR amplification attempts and is attributed to the co-extraction of PCR inhibitors during DNA extraction (Supplementary Figure 2, lanes 5, 6 and 10). Furthermore, pooling multiple PCR amplifications also did not improve the resolution of these samples, as the maximum loading capacity of each well was limited to $60 \mu \mathrm{l}$. The highly individualized communities identified by TGGE suggest microbial diversity is a function of host-specific factors, which appear unrelated to weight status. No distinct microbial communities could be ascribed as unique to either group, suggesting caution in applying community profiling to the search for a community-associated obesity marker.

Fecal SCFA concentration differences between obese and normal-weight children

Concentrations of SCFA present in feces of obese and normal-weight children were determined using HPLC
Table 3 Bacterial populations measured by qPCR in fecal samples from obese $(n=15)$ and normal-weight children $(n=15)$

\begin{tabular}{|c|c|c|c|c|c|}
\hline \multirow{2}{*}{$\begin{array}{l}\text { Bacterial phylogeny } \\
\text { population }\end{array}$} & \multicolumn{2}{|c|}{ Obese } & \multicolumn{2}{|c|}{ Normal-weight } & \multirow[t]{2}{*}{ P-value } \\
\hline & $\begin{array}{c}\log _{10} \\
\text { copies- } 1 g^{a}\end{array}$ & s.d. & $\begin{array}{c}\log _{10} \\
\text { copies- } 1 g^{\mathrm{a}}\end{array}$ & s.d. & \\
\hline Eubacteria & 10.8 & \pm 0.9 & 10.7 & \pm 0.6 & 0.61 \\
\hline \multicolumn{6}{|l|}{ Bacteroidetes } \\
\hline Bacteroides & 9.8 & \pm 0.5 & 10.0 & \pm 0.6 & 0.34 \\
\hline \multicolumn{6}{|l|}{ Actinobacteria } \\
\hline Bifidobacterium & 7.6 & \pm 0.7 & 7.3 & \pm 0.5 & 0.24 \\
\hline \multicolumn{6}{|l|}{ Proteobacteria } \\
\hline Enterobacteriaceae & 8.1 & \pm 1.2 & 7.7 & \pm 0.4 & 0.20 \\
\hline Firmicutes & 9.5 & \pm 0.8 & 9.3 & \pm 0.5 & 0.13 \\
\hline \multicolumn{6}{|l|}{ Firmicutes/bacilli } \\
\hline Lactobacillus & 6.1 & \pm 0.7 & 6.8 & \pm 0.9 & 0.19 \\
\hline \multicolumn{6}{|c|}{ Firmicutes/clostridial cluster XIVa } \\
\hline Roseburia/E. rectale & 9.8 & \pm 0.6 & 9.4 & \pm 0.6 & 0.26 \\
\hline \multicolumn{6}{|c|}{ Firmicutes/clostridial cluster IV } \\
\hline F. prausnitzii & 8.4 & \pm 0.6 & 7.9 & \pm 0.9 & 0.19 \\
\hline Sulfate reducing bacteria & 6.6 & \pm 1.4 & 6.4 & \pm 1.3 & 0.51 \\
\hline
\end{tabular}

Abbreviation: s.d., standard deviations of the mean. ${ }^{\mathrm{a}}$ Data are mean $\log _{10}$ copies $16 \mathrm{~S} \mathrm{rRNA} / \mathrm{g}$ feces.

(Table 4). Fecal metabolite analyses represent endpoint measurements of metabolic activity within the human large intestine as both the number of active enteric bacteria in the small intestine and the absence of SCFA ingested through foods limit SCFA in the small intestine. ${ }^{23}$ SCFA are produced as a function of microbial fermentation of non-absorbed or non-digestible carbohydrates with host metabolism of these compounds occurring after epithelial uptake. Significantly higher concentrations of butyrate and propionate $(P<0.05)$ were detected in obese ( $33.1 \mathrm{~mm}$ and $29.2 \mathrm{mM}$, respectively) versus normal-weight children $(25.3 \mathrm{~mm}$ and $21.6 \mathrm{~mm}$, respectively). Total SCFA concentrations were not statistically different. In general, large individual intragroup variations were observed, with variation coefficients between 20 and $45 \%$ for metabolite concentrations and ratios. Concentrations of acetate were similar between the two groups (Table 4). Analysis of SCFA ratios revealed no statistically relevant differences in acetate/butyrate, acetate/ propionate or butyrate/propionate ratios in obese and normal-weight children. Ratios of individual SCFA/total SCFA were also similar between both groups (Table 4).

\section{Intermediate metabolites present in obese and normal-weight children}

Both the presence and concentrations of various intermediate metabolites were determined by HPLC (Table 5). Only trace amounts of lactose were detected in both groups, suggesting complete enzymatic hydrolysis of lactose to glucose and galactose. Concentrations of the branched chain fatty acids isovalerate were similar in obese $(2.18 \mathrm{~mm})$ and normal-weight children $(3.05 \mathrm{mM})$, whereas isobutyrate was significantly higher in obese children (Table 5). Higher concentrations of formate were detected in the obese group; 
Table 4 SCFA concentrations and ratios of fecal samples from obese and normal-weight children assessed by HPLC

\begin{tabular}{|c|c|c|c|c|c|c|c|}
\hline \multirow[t]{2}{*}{ Metabolite } & \multicolumn{3}{|c|}{ Obese } & \multicolumn{3}{|c|}{ Normal-weight } & \multirow[t]{2}{*}{ P-value } \\
\hline & $m M^{\mathrm{a}}$ & s.d. & $\%$ SCFA & $m M^{\mathrm{a}}$ & s.d. & $\%$ SCFA & \\
\hline Total SCFA & 142.8 & \pm 28.6 & 100 & 124.9 & \pm 31.5 & 100 & 0.09 \\
\hline Acetate & 80.6 & \pm 18.8 & 56.4 & 77.9 & \pm 24.6 & 62.3 & 0.97 \\
\hline Butyrate & 33.1 & \pm 10.3 & 23.2 & 25.3 & \pm 8.7 & 20.4 & $0.01^{*}$ \\
\hline Propionate & 29.2 & \pm 13.5 & 20.4 & 21.6 & \pm 8.2 & 17.3 & $0.01^{*}$ \\
\hline Metabolic ratio & Mean ratio ${ }^{a}$ & & s.d. & Mean ratio $^{\mathrm{a}}$ & & s.d. & P-value \\
\hline Acetate/butyrate & $2.78: 1$ & & \pm 0.83 & $3.10: 1$ & & \pm 0.75 & 0.39 \\
\hline Acetate/propionate & $3.64: 1$ & & \pm 0.75 & $3.40: 1$ & & \pm 0.80 & 0.35 \\
\hline Butyrate/propionate & $1.25: 1$ & & \pm 0.64 & $1.14: 1$ & & \pm 0.28 & 0.53 \\
\hline Total SCFA/acetate & $1.77: 1$ & & \pm 0.19 & $1.65: 1$ & & \pm 0.13 & 0.05 \\
\hline Total SCFA/butyrate & 4.38:1 & & \pm 1.02 & $5.03: 1$ & & \pm 0.88 & 0.06 \\
\hline Total SCFA/propionate & $5.36: 1$ & & \pm 1.43 & $5.54: 1$ & & \pm 0.93 & 0.67 \\
\hline
\end{tabular}

Abbreviations: HPLC, high-pressure liquid chromatography; SCFA, short-chain fatty acid; s.d., standard deviation. ${ }^{\mathrm{a} D a t a}$ are mean values calculated for fecal samples of each group (obese: $n=15$; normal-weight: $n=15$ ). Mean SCFA concentrations that are significantly different between obese and normal-weight children are denoted by $* P<0.05$.

Table 5 Intermediate metabolite concentrations of fecal samples from obese and normal-weight children assessed by HPLC

\begin{tabular}{|c|c|c|c|c|c|}
\hline \multirow[t]{2}{*}{ Metabolite } & \multicolumn{2}{|c|}{ Obese } & \multicolumn{2}{|c|}{ Normal-weight } & \multirow[t]{2}{*}{ P-value } \\
\hline & $m M^{\mathrm{a}}$ & s.d. & $m M^{\mathrm{a}}$ & s.d. & \\
\hline Lactose & 0.01 & \pm 0.04 & 0.07 & \pm 0.17 & 0.15 \\
\hline Glucose & 4.28 & \pm 5.23 & 26.61 & \pm 61.73 & 0.11 \\
\hline Lactate & 0.80 & \pm 0.77 & 12.02 & \pm 14.3 & $0.009^{*}$ \\
\hline Formate & 1.22 & \pm 4.73 & 0.24 & \pm 1.12 & 0.44 \\
\hline Isobutyrate & 7.87 & \pm 2.7 & 3.11 & \pm 2.11 & $0.0007^{*}$ \\
\hline Isovalerate & 2.33 & \pm 2.45 & 3.05 & \pm 2.33 & 0.38 \\
\hline Valerate & 0.97 & \pm 1.27 & 2.09 & \pm 1.56 & $0.02^{*}$ \\
\hline
\end{tabular}

Abbreviations: HPLC, high-pressure liquid chromatography; s.d., standard deviation of the means. ${ }^{a}$ Data are mean values calculated for fecal samples of each group (obese: $n=15$; normal-weight: $n=15$ ). Intermediate metabolite concentrations that are significantly different are denoted by ${ }^{*} P<0.05$.

however, the difference was not statistically significant. Concentrations of lactate were significantly higher in normal-weight children $(P<0.05)$. Valerate was also present at significantly higher amounts $(P<0.05)$ in normal-weight subjects (Table 4). Residual amounts of glucose were detected in both groups, with higher concentrations of glucose (26.1 mM) measured in normal-weight versus obese subjects (4.55 mM). However, the large s.d. calculated for the normalweight results $( \pm 61.71 \mathrm{mM})$ suggests cautionary interpretation of these results. When glucose calculations were corrected for by removal of several high outlying values, both the mean and s.d. were reduced (3.3 $\mathrm{mm}$ and $4.3 \mathrm{~mm}$, respectively) and the difference between the obese and normal-weight group became statistically insignificant.

\section{Discussion}

In the present study, we analyzed gut microbial compositions and fecal metabolite concentrations in obese and normal-weight children from a heterogeneous population that included the majority of ethnicities present in Germanspeaking Switzerland. Dietary intakes were not extensively recorded and not controlled for in this study, as our goal was to randomly sample obese and normal-weight children exhibiting normal eating and lifestyle behavior. To our knowledge, this represents the first description of the composition and metabolite characterization of gut microbiota obtained from obese and normal-weight children sampled without dietary restrictions or controls.

Community membership of gut microbiota from obese and normal-weight children was highly similar. Of the major microbial phyla and genera present in feces, no significant quantitative differences in individual communities of obese and normal-weight gut microbiota could be identified. Microbiota were predominately composed of Firmicutes and Bacteroides. Roseburia/E. rectale, a member of Clostridia cluster XIVa, represents a larger Firmicutes subgroup in comparison with cluster IV of which F. prausnitzii is a member. Hence, the higher proportion of Roseburia/E. rectale/ F. prausnitzii detected in both groups is expected. Although the Bacteroidetes phylum comprises several genera in addition to Bacteroides, the Bacteroides genus has been demonstrated to be one of four major groups of adult microbiota. ${ }^{24,25}$ In contrast to adult studies in which a higher Firmicutes/Bacteroidetes ratio has been proposed as relevant in the development of obesity, no correlation of an increased Firmicutes/Bacteroides ratio to childhood obesity could be established., ${ }^{4-9}$ Community profiling of individual obese and normal-weight subjects by TGGE illustrated highly variable and individual biodiversity. Host-specific uniqueness in microbial diversity was sufficiently demonstrated by TGGE profiling and is in agreement with previously published reports. ${ }^{26,27}$ Intergroup inspection of microbial diversity failed to demonstrate any communities associated exclusively with weight status. 
Higher levels of SCFA have been reported in obese versus normal-weight adult subjects, although the cause and consequence of these results and its relationship to obesity remain unclear. ${ }^{11}$ Analysis of fecal metabolites in this study identified a tendency of obese gut microbiota in producing higher levels of SCFA as previously reported. Significantly higher levels of butyrate were detected in obese children, despite similar acetate concentrations observed in each group (Table 4). Multiple pathways dedicated to butyrate production are enzymatically catalyzed through various mechanisms specific to butyrate-producing species in the large intestine. Conversion of acetate to butyrate, catalyzed by butyryl coA/acetate coA transferase, represents the major pathway for butyrate production. ${ }^{28}$ The similar acetate/ butyrate ratio in obese and normal-weight children indicates that more efficient conversion of acetate to butyrate is not the likely predominating mechanism responsible for increased butyrate concentrations in obese children. Butyrate kinase activity represents a second possible mechanism for butyrate production, although distribution of the butyrate kinase gene is more taxonomically restricted, predominately present in members of clostridia. ${ }^{28}$ Little information exists regarding regulation of butyrate-producing pathways in gut microbiota, suggesting that the increased concentrations of butyrate detected in obese children could be a function of differential induction and utilization of butyrate-producing pathways.

A third putative route of butyrate production involves conversion of lactate by lactate-utilizing butyrate-producing bacteria. This activity has been reported in clostridial cluster XIVa species Eubacterium hallii and Anaerostipes caccae but is absent from $R$. intestinalis and E. rectale. ${ }^{29}$ Lactate is the major fermentation product of lactic acid bacteria, including lactobacilli, streptococci and enterococci and bifidobacteria, although lactate production has also been reported in strict anaerobes related to Eubacterium spp. ${ }^{30,31}$ Despite the many species capable of intestinal lactate production, lactate is normally not detected in feces. The presence of lactate in normal-weight, but not obese children, and significantly higher concentrations of butyrate in obese children suggests further the presence of lactate-utilizing butyrate-producing species in obese children (Tables 4 and 5). It is unlikely that the lactate detected in normal-weight feces was a result of intestinal disease as no subjects reported any abnormal gastrointestinal functioning.

A further possible route of lactate consumption involves oxidation by SRB to produce $\mathrm{H}_{2} \mathrm{~S}$. SRB were demonstrated to effectively compete with lactate-utilizing butyrate producers in in vitro fermentations. ${ }^{32}$ SRB were not significantly different between obese and normal-weight children; however, individual populations demonstrated the highest variation of any bacterial population tested (Table 3). SRB populations in both healthy and ulcerative colitis patients were also not significantly different, although total viable SRB counts have been correlated to the severity of the disease. ${ }^{33,34}$ Lactate metabolism by SRB generates $\mathrm{H}_{2}$ as an electron sink during abundant substrate concentrations. High colonic $\mathrm{H}_{2}$ partial pressure inhibits fermentation by preventing regeneration of $\mathrm{NADH}$ via the action of NADH/ ferredoxin oxidoreductase and hydrogenase. Reduction of $\mathrm{H}_{2}$ partial pressure in the intestine is crucial for sustaining fermentative processes and is facilitated by oxidation during methanogenesis, acetogenesis, but also during dissimilatory sulfate reduction as SRB may utilize both endogenously produced and exogenous $\mathrm{H}_{2}$ as electron donor. The individual nature of SRB viability and to what extent this may potentially impact enteric metabolic function, warrants further investigation.

Significantly higher levels of propionate were also identified in obese children (Table 4). Propionate-producing species are more restricted in taxonomic distribution in comparison with the abundance of butyrate-producing species, with two major pathways for propionate production having been described. Members of the Gram-negative Bacteroides-Prevotella group produce propionate via succinate or fumarate, whereas Gram-positive species of clostridial cluster IX convert lactate to propionate via the acrylate pathway. ${ }^{35,36}$ In vitro analysis of lactate metabolism during fermentation revealed it is primarily converted to butyrate and not propionate. ${ }^{37}$ Thus, it is unlikely that the depletion of lactate observed in obese children significantly contributed to the higher propionate concentrations. Neither succinate nor fumarate were measured in this study, thus, the contribution of propionate formed via this pathway cannot be determined.

In vitro gut fermentation modeling of obese and normalweight child microbiota presents a possible route for distinguishing endpoint metabolic measurements from active metabolism. We are currently evaluating multiple in vitro gut fermentation models using obese and normalweight microbiota to better elucidate factors determining microbial metabolic activity. As the metabolic fate of acetate, butyrate and propionate has a profound effect on human health, understanding the exact mechanisms governing their production to better modulate enteric metabolite levels is needed. Several studies have effectively demonstrated significant differences between groups using cohorts similar in size, confirming our sample size as sufficiently large enough to detect any significant differences between obese and normal-weight communities had they existed. ${ }^{4,38,39}$ Our results suggest that a dysbiosis may be involved in the etiology of childhood obesity. In turn, aberrant and overactive metabolic activity within the intestine could dictate survival or loss of individual microbial communities. This hypothesis suggests that the increased Fimicutes/Bacteroidetes ratio observed in obese adults could be a result of dysbiosis arising from adaptation of individual microbial communities to long-term metabolic dysfunction. Validation of this hypothesis could be achieved by evaluating gut microbial compositions and metabolite concentrations of adolescents, the missing link between childhood and adult results. Metabolic profiling using 'top down' systems biology 
approaches of all three study populations (i.e., children, adolescents and adults) could unravel the exact host (e.g., genotype and phenotype) and microbial (e.g., metatranscriptome) factors determining evolution of the 'metabotype' and to what extent the metabolome dictates community structure. Such studies have been used to investigate differences in drug development and are becoming increasingly of interest in developing personalized nutrition and healthcare. ${ }^{40}$ To our knowledge, no studies following obese children into adulthood have been performed to monitor the changes in gut microbial populations and metabolic activities in the same individual over time. Our results present compelling evidence for the usefulness of such studies in unraveling the cause and consequence relationship between gut microbiota and obesity.

\section{Conflict of interest}

The authors declare no conflict of interest.

\section{Acknowledgements}

We thank Dr Isabelle Aeberli for volunteer recruitment and data analysis. This work was supported by the ETHIIRA grant ETH-18 08-02 and the Swiss Foundation for Nutrition Research.

\section{References}

1 Cummings JH, Macfarlane GT. Colonic microflora: nutrition and health. Nutrition 1997; 13: 476-478.

2 Backhed F, Ley RE, Sonnenburg JL, Peterson DA, Gordon JI. Hostbacterial mutualism in the human intestine. Science 2005; 307: 1915-1920.

3 Elson CO, Sartor RB, Tennyson GS, Riddell RH. Experimental models of inflammatory bowel disease. Gastroenterology 1995; 109: 1344-1367.

4 Ley RE, Backhed F, Turnbaugh P, Lozupone CA, Knight RD, Gordon JI. Obesity alters gut microbial ecology. Proc Natl Acad Sci USA 2005; 102: 11070-11075.

5 Sartor RB. The influence of normal microbial flora on the development of chronic mucosal inflammation. Res Immunol 1997; 148: 567-576.

6 Nadal I, Santacruz A, Marcos A, Warnberg J, Garagorri M, Moreno LA et al. Shifts in clostridia, bacteroides and immunoglobulin-coating fecal bacteria associated with weight loss in obese adolescents. Int J Obes 2009; 33: 758-767.

7 Eckburg PB, Bik EM, Bernstein CN, Purdom E, Dethlefsen L, Sargent $\mathrm{M}$ et al. Diversity of the human intestinal microbial flora. Science 2005; 308: 1635-1638.

8 Ley RE, Turnbaugh PJ, Klein S, Gordon JI. Microbial ecology: human gut microbes associated with obesity. Nature 2006; 444: 1022-1023.

9 Turnbaugh PJ, Ley RE, Mahowald MA, Magrini V, Mardis ER, Gordon JI. An obesity-associated gut microbiome with increased capacity for energy harvest. Nature 2006; 444: 1027-1031.
10 Duncan SH, Lobley GE, Holtrop G, Ince J, Johnstone AM, Louis P et al. Human colonic microbiota associated with diet, obesity and weight loss. Int J Obes (Lond) 2008; 32: 1720-1724.

11 Schwiertz A, Taras D, Schafer K, Beijer S, Bos NA, Donus C et al. Microbiota and SCFA in lean and overweight healthy subjects. Obesity (Silver Spring) 2010; 18: 190-195.

12 Robert C, Bernalier-Donadille A. The cellulolytic microflora of the human colon: evidence of microcrystalline cellulose-degrading bacteria in methane-excreting subjects. FEMS Microbiol Ecol 2003; 46: 81-89.

13 McNeil NI. The contribution of the large intestine to energy supplies in man. Am J Clin Nutr 1984; 39: 338-342.

14 Scheppach W. Effects of short chain fatty acids on gut morphology and function. Gut 1994; 35 (1 Suppl): S35-S38.

15 Backhed F, Ding H, Wang T, Hooper LV, Koh GY, Nagy A et al. The gut microbiota as an environmental factor that regulates fat storage. Proc Natl Acad Sci USA 2004; 101: 15718-15723.

16 Flint HJ, Bayer EA, Rincon MT, Lamed R, White BA. Polysaccharide utilization by gut bacteria: potential for new insights from genomic analysis. Nat Rev Microbiol 2008; 6: 121-131.

17 Aeberli I, Molinari L, Spinas G, Lehmann R, l'Allemand D, Zimmermann MB. Dietary intakes of fat and antioxidant vitamins are predictors of subclinical inflammation in overweight Swiss children. Am J Clin Nutr 2006; 84: 748-755.

18 Bryant MP. Commentary on the Hungate technique for culture of anaerobic bacteria. Am J Clin Nutr 1972; 25: 1324-1328.

19 Duncan SH, Hold GL, Harmsen HJ, Stewart CS, Flint HJ. Growth requirements and fermentation products of Fusobacterium prausnitzii, and a proposal to reclassify it as Faecalibacterium prausnitzii gen. nov., comb. nov. Int J Syst Evol Microbiol 2002; 52 (Part 6): 2141-2146.

20 Ogier JC, Son O, Gruss A, Tailliez P, Delacroix-Buchet A. Identification of the bacterial microflora in dairy products by temporal temperature gradient gel electrophoresis. Appl Environ Microbiol 2002; 68: 3691-3701.

21 Muyzer G, de Waal EC, Uitterlinden AG. Profiling of complex microbial populations by denaturing gradient gel electrophoresis analysis of polymerase chain reaction-amplified genes coding for 16S rRNA. Appl Environ Microbiol 1993; 59: 695-700.

22 Cleusix V, Lacroix C, Vollenweider S, Le Blay G. Glycerol induces reuterin production and decreases Escherichia coli population in an in vitro model of colonic fermentation with immobilized human feces. FEMS Microbiol Ecol 2008; 63: 56-64.

23 Binder HJ. Role of colonic short-chain fatty acid transport in diarrhea. Annu Rev Physiol 2010; 72: 297-313.

24 Harmsen HJM, Raangs GC, He T, Degener JE, Welling GW. Extensive set of $16 \mathrm{~S}$ rRNA-based probes for detection of bacteria in human feces. Appl Environ Microb 2002; 68: 2982-2990.

25 Rigottier-Gois L, Le Bourhis AG, Gramet G, Rochet V, Dore J. Fluorescent hybridisation combined with flow cytometry and hybridisation of total RNA to analyse the composition of microbial communities in human faeces using $16 \mathrm{~S}$ rRNA probes. FEMS Microbiol Ecol 2003; 43: 237-245.

26 Zoetendal EG, Akkermans ADL, De Vos WM. Temperature gradient gel electrophoresis analysis of 16S rRNA from human fecal samples reveals stable and host-specific communities of active bacteria. Appl Environ Microb 1998; 64: 3854-3859.

27 Zoetendal EG, Vaughan EE, de Vos WM. A microbial world within us. Mol Microbiol 2006; 59: 1639-1650.

28 Louis P, Duncan SH, McCrae SI, Millar J, Jackson MS, Flint HJ. Restricted distribution of the butyrate kinase pathway among butyrate-producing bacteria from the human colon. J Bacteriol 2004; 186: 2099-2106.

29 Duncan SH, Louis P, Flint HJ. Lactate-utilizing bacteria, isolated from human feces, that produce butyrate as a major fermentation product. Appl Environ Microb 2004; 70: 5810-5817.

30 Barcenilla A, Pryde SE, Martin JC, Duncan SH, Stewart CS, Henderson $\mathrm{C}$ et al. Phylogenetic relationships of butyrate-producing bacteria from the human gut. Appl Environ Microb 2000; 66: 1654-1661. 
31 Hobson PN, Summers R, Owen EC, Spencer JC, West DW. A study of cultures of rumen anaerobic bacteria in presence of excess riboflavine (vitamin B2). Proc Nutr Soc 1969; 28: A53.

32 Marquet P, Duncan SH, Chassard C, Bernalier-Donadille A, Flint HJ. Lactate has the potential to promote hydrogen sulphide formation in the human colon. FEMS Microbiol Lett 2009; 299: 128-134.

33 Zinkevich V, Beech IB. Screening of sulfate-reducing bacteria in colonoscopy samples from healthy and colitic human gut mucosa. FEMS Microbiol Ecol 2000; 34: 147-155.

34 Pitcher MCL, Beatty ER, Cummings JH. The contribution of sulphate reducing bacteria and 5-aminosalicylic acid to faecal sulphide in patients with ulcerative colitis. Gut 2000; 46: 64-72.

35 Bernalier A, Dore J, Durand M. Biochemistry of fermentation. In: Gibson GR and Roberfroid, MB (eds). Colonic Microbiota, Fermentation and Health. Kluwer Academic Publishers: NL, 1999, pp 37-53.

36 Macfarlane GT, Gibson GR. Carbohydrate fermentation, energy transduction and gas metabolism in the human large intestine. In: Mackie RI and White BA (eds). Gastrointestinal Microbiology. Chapman and Hall: London, 1997, pp 269-318.

37 Bourriaud C, Robins RJ, Martin L, Kozlowski F, Tenailleau E, Cherbut $\mathrm{C}$ et al. Lactate is mainly fermented to butyrate by human intestinal microfloras but inter-individual variation is evident. J Appl Microbiol 2005; 99: 201-212.

38 Kalliomaki M, Collado MC, Salminen S, Isolauri E. Early differences in fecal microbiota composition in children may predict overweight. Am J Clin Nutr 2008; 87: 534-538.

39 Walker AW, Ince J, Duncan SH, Webster LM, Holtrop G, Ze X et al. Dominant and diet-responsive groups of bacteria within the human colonic microbiota. ISME J 2011; 5: 220-230.

40 Holmes E, Wilson ID, Nicholson JK. Metabolic phenotyping in health and disease. Cell 2008; 134: 714-717.
41 Guo X, Xia X, Tang R, Zhou J, Zhao H, Wang K. Development of a real-time PCR method for Firmicutes and Bacteroidetes in faeces and its application to quantify intestinal population of obese and lean pigs. Lett Appl Microbiol 2008; 47: 367-373.

42 Ramirez-Farias C, Slezak K, Fuller Z, Duncan A, Holtrop G, Louis P. Effect of inulin on the human gut microbiota: stimulation of Bifidobacterium adolescentis and Faecalibacterium prausnitzii. Br J Nutr 2009; 101: 541-550.

43 Furet JP, Firmesse O, Gourmelon M, Bridonneau C, Tap J, Mondot $\mathrm{S}$ et al. Comparative assessment of human and farm animal faecal microbiota using real-time quantitative PCR. FEMS Microbiol Ecol 2009; 68: 351-362.

44 Cleusix V, Lacroix C, Dasen G, Leo M, Le Blay G. Comparative study of a new quantitative real-time PCR targeting the xylulose5-phosphate/fructose-6-phosphate phosphoketolase bifidobacterial gene $(\mathrm{xfp})$ in faecal samples with two fluorescence in situ hybridization methods. J Appl Microbiol 2010; 108: 181-193.

45 Frank DN, Amand ALS, Feldman RA, Boedeker EC, Harpaz N, Pace NR. Molecular-phylogenetic characterization of microbial community imbalances in human inflammatory bowel diseases. Proc Natl Acad Sci USA 2007; 104: 13780-13785.

46 Pereyra LP, Hiibel SR, Riquelme MVP, Reardon KF, Pruden A. Detection and quantification of functional genes of cellulosedegrading, fermentative, and sulfate-reducing bacteria and methanogenic archaea. Appl Environ Microb 2010; 76: 2192-2202.

This work is licensed under the Creative Commons Attribution-NonCommercial-No Derivative Works 3.0 Unported License. To view a copy of this license, visit http://creativecommons.org/ licenses/by-nc-nd/3.0/

Supplementary Information accompanies the paper on the Nutrition and Diabetes website (http://www.nature.com/nutd) 